\title{
LA PRODUCCIÓN CERÁMICA VILUCO ENTRE LOS SIGLOS XV Y XVII (PROVINCIA DE MENDOZA, ARGENTINA)
}

\author{
VILUCO POTTERY PRODUCTION BETWEEN THE XVth AND XVIIth \\ CENTURIES (PROVINCIA DE MENDOZA, ARGENTINA)
}

\author{
Cristina Prieto ${ }^{1}$ y Horacio Chiavazza ${ }^{2}$
}

\begin{abstract}
Se presentan los estudios sobre la variabilidad alfarera de la Cultura Viluco (Centro-Oeste argentino). La Cultura Viluco ocupó el norte y centro de Mendoza, y el sur de San Juan, entre el siglo XV y mediados del XVII, cuando se suscitaron profundos cambios en las poblaciones locales debido a la expansión del imperio Inka y luego del español. La producción cerámica fue sensible a estos cambios, por lo cual proponemos que se desarrollaron dos modos de producción alfarera: por un lado, durante el período de dominación Inka en algunos sitios se produjo de forma centralizada y, por otro, luego de la llegada de los españoles la producción fue descentralizada y centralizada. Esto último debido a la incorporación de la tecnología del torno y de nuevas formas cerámicas.

Palabras claves: Centro-Oeste argentino, Cultura Viluco, producción alfarera, cerámica.

In this paper we present studies of variability in the pottery of the Viluco Culture (midwestern Argentina). Viluco Culture spread over northern and central Mendoza, and south of San Juan, between the XVth and XVIIth centuries, with the social and cultural changes produced by the expansion of the Inka and Spanish empires. In these contexts pottery production changed. We propose two modes of pottery production: a centralized production during the Inka domination period at some sites; and with the arrival of the Spaniards the production became descentralized and recentralized. The latter due to the implementation of new technology, the wheel, and new ceramic shapes.
\end{abstract}

Key words: Middle West of Argentina, Viluco Culture, pottery production, ceramics.

En este trabajo presentamos los resultados del análisis de cerámica Viluco obtenida de un contexto doméstico de las Ruinas de San Francisco (RSF en adelante) y de diversos sitios funerarios $\mathrm{y}$ algunos hallazgos aislados, del norte y centro de la Provincia de Mendoza. Este material es el más abundante y diagnóstico de esta cultura y su variabilidad nos ha llevado a estudiarlo con una metodología que permitiera interrelacionar y potenciar la información cerámica, explorando aspectos ligados a la producción. Por otro lado, la consulta de estudios etnohistóricos ha sido fundamental para comprender cómo percibió una parte de la población hispana a los grupos indígenas locales y nos ayuda complementariamente $-\mathrm{y}$ no para contrastar la información arqueológica- a explorar algunos aspectos del desarrollo de estos grupos en el período de contacto.

\section{Antecedentes}

La Cultura Viluco (Lagiglia 1978) se desarrolló en el norte y centro de la Provincia de Mendoza y Sur de San Juan (Argentina), parte importante de la Subárea Centro-Oeste argentino. Cronológicamente ha sido ubicada a mediados del siglo XV hasta mediados del XVII (Bárcena 1998), aunque existe un fechado sobre carbón más antiguo (Chiavazza 1995). Se han realizado varias propuestas en torno al origen de Viluco: el origen preinkaico (Chiavazza 1995; Lagiglia 1978); el origen inkaico (García 1999); el origen poshispánico (Michieli 1998). Consideramos la posibilidad de un origen preinkaico, ya que los análisis de TL permiten localizar las fechas más tempranas entre mediados del siglo XV y mediados del XVI, incluso hay un fechado radiocarbónico preinkaico en el sitio Rincón de los Helados: componente RH2

\footnotetext{
Centro de Investigaciones Ruinas de San Francisco. Ituzaingó 2134 (5500), Mendoza, Argentina. cristiprieto@ gmail.com

2 Facultad de Filosofía y Letras, Universidad Nacional de Cuyo, Ciudad Universitaria Parque General San Martín s/n (5500) Mendoza, Argentina. Museo del Área Fundacional de Mendoza, Plaza Pedro del Castillo s/n, Mendoza, Argentina. hchiavazza@gmail.com
} 
$610 \pm 80$ a.p. (LP-642 Latyr) (Chiavazza 1995). De todos modos queda aún un vacío por explicar: su relación con Agrelo, cultura arqueológica previa a Viluco. Los fechados de contacto hispano-indígena provienen de diversos contextos, uno de carbón de RSF 440 \pm 40 a.p. (URU0279; Chiavazza y Prieto 2001); y varios TL, como las del Cabildo y el cementerio de Viluco (Bárcena 1998) y de Capiz I (Durán y Novellino 2003) ${ }^{1}$. Estos fechados permitirían relacionar la Cultura Viluco con los Huarpes etnohistóricos.

Respecto a la dominación inkaica, ésta se habría producido durante el reinado de Tupac Inka Yupanqui, pero su materialización se habría concretado en el de Huayna Cápac, en el lapso aproximado de los años 1471 y 1525/1527. Se ha postulado que la dominación en el suroeste de San Juan y noroeste de Mendoza habría sido funcional a la conquista inkaica en los valles centrales y Norte Chico de Chile (Bárcena 1994; Bárcena y Román 1990; Parisii 1994). Esta se habría materializado a partir de la introducción de mitmaqkuna trasladado desde el Norte Chico chileno (Bárcena 1994; Sacchero y García 1991). Específicamente sobre la presencia inka en los valles bajos hay evidencia documental de al menos un sitio en el valle de Huentota, donde se habría cultivado para abastecer los sitios anexos a la red vial (Bárcena 1994; Parisii 1994) ${ }^{2}$, pero sin que se diera el dominio efectivo del valle (Parisii 1994). Una evidencia interesante sobre la organización de la producción alfarera durante este período es el uso de la mano de obra indígena local por parte del grupo dominante para la producción cerámica en el tambo de Tambillos (Bárcena y Román 1990). Por su parte, en recientes investigaciones se incluye al valle de Uco en la región de producción, distribución y consumo de bienes dentro de la economía política inka, debido a la incorporación de grupos del Diaguita del Norte Chico chileno asentados por el inka (Ots 2007).

La dominación hispana se concretó con la fundación de Mendoza en 1561 (hay antecedentes de presencia española desde 1551), lo que terminó por desarticular sociopolíticamente a los grupos Huarpes (Parisii 1998; Prieto 2000). La aplicación del sistema de encomienda en 1552 disminuyó la población masculina y se produjo la apropiación definitiva de las tierras irrigadas y de la fuerza laboral indígena. Tanto la apropiación de la tierra como la tributación en tiempos históricos habrían tenido su base y éxito en el sistema de mita impuesto por la anterior dominación inkaica. Este proceso continuo de desarticulación social habría tenido consecuencias en la organización social huarpe que se define con una base cognática de parentesco y bilocalidad (Parisii 1998).

El sitio Ruinas de San Francisco se ubica en el valle de Mendoza, área donde se fundó la ciudad de Mendoza, la que habría tenido el mayor asentamiento Huarpe en el siglo XVI a la llegada de los españoles. A partir de la evidencia documental se ha postulado que aquí las distintas parcialidades encabezadas por sus respectivos caciques practicaban la agricultura, probablemente la ganadería de la llama e intercambiaban con las parcialidades de otros ambientes como los Huarpes de las lagunas de Guanacache y los Puelches del sur (Michieli 1983; Prieto 2000). En el sitio destaca una secuencia ocupacional continua de dos mil años, pasando por las ocupaciones Viluco, jesuita y franciscana hasta su derrumbe con el terremoto de 1861. En este estudio analizamos toda la cerámica Viluco del sitio y destacamos el contexto denominado Crucero Fogón ${ }^{3}$, ya que representa un excepcional contexto primario de una unidad doméstica Viluco en el valle de Mendoza ${ }^{4}$ (Prieto 2005).

\section{Los Estudios Cerámicos}

La diversidad cerámica observada en RSF y considerando que las tipologías han sido elaboradas casi exclusivamente a base de la evidencia de contextos funerarios, nos motivó a desarrollar una línea de investigación cuyo objetivo fundamental es organizar la información de la cerámica Viluco para caracterizar el conjunto alfarero proveniente de diversos contextos, explorando los modos de producción alfarera. La metodología aplicada nos permitió interrelacionar las variables tecnológica, morfológica, funcional y decorativa de los fragmentos (RSF), con las de las vasijas enteras y reconstruidas provenientes de contextos funerarios y algunos hallazgos aislados (Figura 1). La información de las vasijas enteras se usó como referente en la reconstrucción hipotética de formas y para el estudio de los patrones decorativos. Encaramos la investigación a partir de la variabilidad alfarera que se encuentra en cada uno de los aspectos inherentes a la alfarería (tecnología, morfología y decoración) y permite obtener información sobre múltiples aspectos sociales, que van desde el individuo implicado en la manufactura hasta el grupo social en el cual 


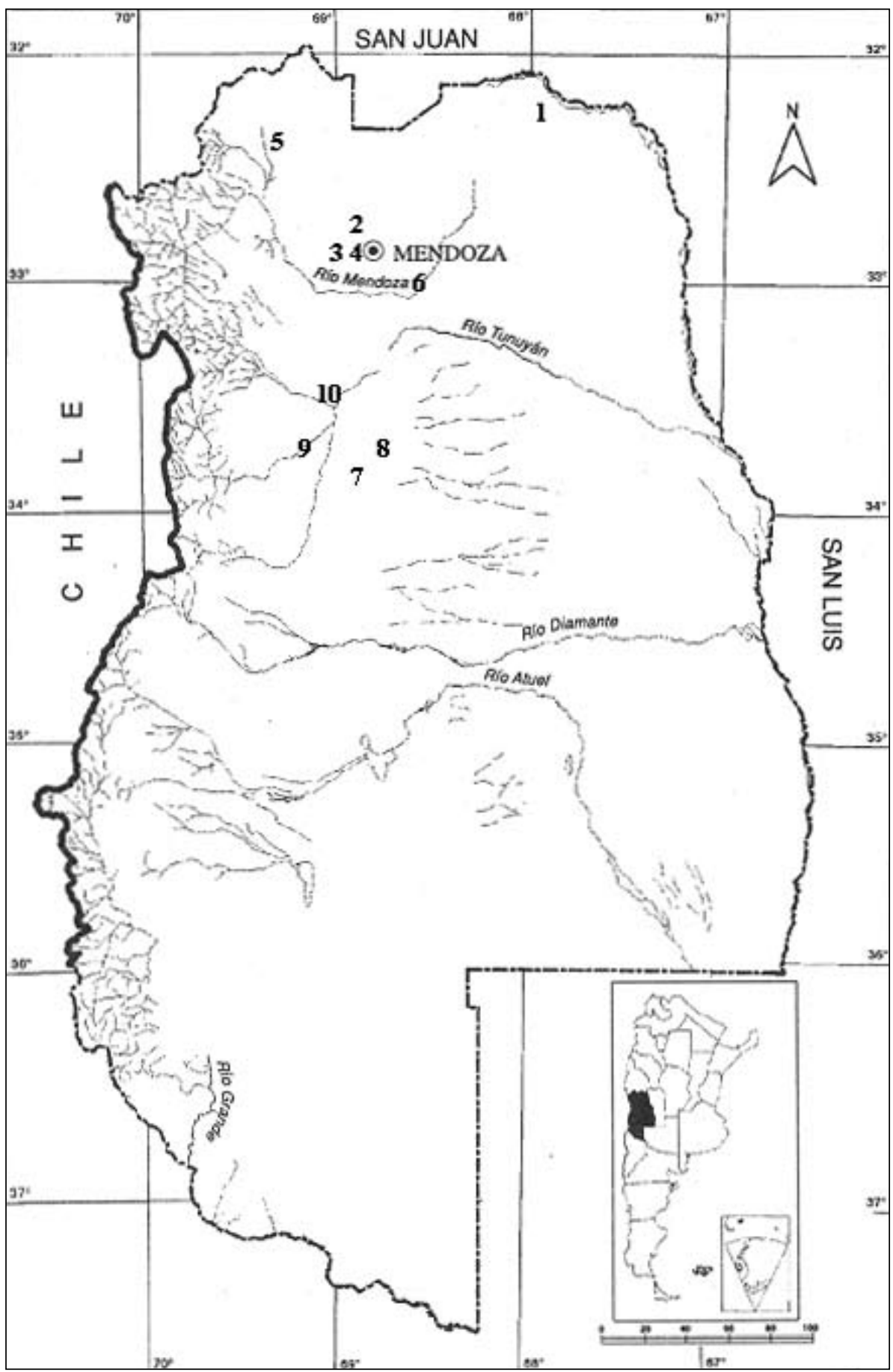

Figura 1. Ubicación de los sitios analizados: Ciudad de Mendoza Ruinas de San Francisco; (1) El Algarrobito (Lavalle); (2) Calle Olascoaga (Las Heras); (3) Beltrán y Liniers (Godoy Cruz); (4) Tacuarí y 20 de junio (Godoy Cruz); (5) Ranchillos (Las Heras); (6) Barrancas (Maipú); (7) Capiz I (San Carlos); (8) Viluco (San Carlos); (9) Arboleda II (Tupungato); (10) Agua Amarga (Tunuyán) (Prieto 2005:80).

Location of sites analyzed: Ciudad de Mendoza Ruinas de San Francisco; (1) El Algarrobito (Lavalle); (2) Calle Olascoaga (Las Heras); (3) Beltrán and Liniers (Godoy Cruz); (4) Tacuarí and 20 de junio (Godoy Cruz); (5) Ranchillos (Las Heras); (6) Barrancas (Maipú); (7) Capiz I (San Carlos); (8) Viluco (San Carlos); (9) Arboleda II (Tupungato); (10) Agua Amarga (Tunuyán) (Prieto 2005:80). 
circularon las vasijas, introduciéndonos en distintas escalas del fenómeno social y en cada acto consciente y subconsciente involucrado desde su manufactura hasta su desecho (Falabella 1997). Específicamente nos interesa indagar sobre la organización de la producción cerámica, porque se encuentra inserta en los sistemas, político, social y económico y por las presiones y oportunidades que brinda el medio (Costin 1991). De este modo, es posible detectar aspectos ligados a la complejidad sociopolítica: movimientos poblacionales, interacciones políticas, económicas y al intercambio de bienes.

\section{Materiales y Método}

Los fragmentos corresponden a los recuperados de las excavaciones de las RSF, un total de 807 fragmentos. Las piezas enteras y reconstruidas suman un total de $40^{5}$ correspondientes a contextos funerarios: Capiz I, Viluco, Barrancas, Agua Amarga, calle Olascoaga, calles Tacuarí y 20 de Junio; y hallazgos aislados sin referencias contextuales de La Arboleda II, Ranchillos, El Algarrobito, calles Beltrán y Liniers (Tabla 1).

La metodología aplicada se basa en los estudios desarrollados ampliamente en Chile Central ${ }^{6}$. Los análisis morfométricos están orientados a definir aspectos de variabilidad/regularidad. Entendemos que a mayor especialización artesanal existiría mayor regularidad en los parámetros métricos y viceversa; por otra parte el grado de variabilidad fijaría los límites para utilizar los parámetros métricos en el análisis de los fragmentos (Falabella et al. 1993). Primero se hizo el estudio de las vasijas enteras, evaluando la regularidad/variabilidad a partir del análisis de las desviaciones estándar (DS) de cada una de las medidas estudiadas 7 . El análisis se realizó a distintas escalas: análisis de medidas intra-vasija; intra-categoría morfológica; intra-sitio; inter-sitios. A nivel intra-categoría se evaluó la existencia de tamaños modales (categorías de capacidad o volumen), se realizaron histogramas para el volumen (Vol) de cada categoría morfológica y se analizaron las medidas relacionadas con la capacidad de las vasijas: Altura Total (HTotal), Diámetro Máximo (Diám. Máximo) o Diámetro de Borde (Diám. Borde).

A partir de los resultados de las vasijas enteras se hizo el análisis morfométrico de los fragmentos de las RSF. Primero, se evaluó la posibilidad de discriminar categorías morfológicas, a nivel cuantitativo se analizó la distribución de medidas de cada categoría morfológica y cualitativamente se discriminaron los atributos que son únicos en cada categoría morfológica (borde, cuello y base). En segundo lugar, se analizó la posibilidad de realizar predicciones de capacidad (volumen) en las vasijas definidas entre los fragmentos. Para ello se calcularon los coeficientes de correlación con las medidas que pueden ser tomadas entre los fragmentos y se consideró un coeficiente mayor a 0,750 como aceptable (Falabella et al. 1993). La posibilidad de estimar capacidad se evaluó en base a ecuaciones de regresión.

El análisis macroscópico de pastas se hizo con lupa binocular (entre 10X y 60X) sobre cortes frescos del 63\% ( $n=507)$ del total de la muestra. Se definieron patrones de pasta a partir de las características de la matriz (color, cocción, fractura y textura) y los antiplásticos (color, forma, tamaño y densidad $)^{8}$, para finalmente determinar familias de

Tabla 1. Sitios y número de vasijas. Sites and number of vessels.

\begin{tabular}{|c|c|c|c|c|c|c|c|}
\hline Sitio & Dpto. & $\begin{array}{l}\text { Escudilla } \\
\text { (n) }\end{array}$ & $\begin{array}{c}\text { Jarra } \\
(\mathrm{n})\end{array}$ & $\begin{array}{c}\text { Kero } \\
(\mathrm{n})\end{array}$ & $\begin{array}{l}\text { Aribaloide } \\
\text { (n) }\end{array}$ & $\begin{array}{l}\text { Aisana } \\
\text { (n) }\end{array}$ & Total \\
\hline Capiz I & San Carlos & 1 & 1 & 1 & - & - & 3 \\
\hline Viluco & San Carlos & 2 & 4 & 5 & - & - & 11 \\
\hline Arboleda II & Tupungato & & 1 & - & - & - & 1 \\
\hline Agua Amarga & Tupungato & 3 & 4 & 3 & 1 & 1 & 12 \\
\hline Barrancas & Maipú & 1 & 3 & 1 & - & - & 5 \\
\hline Calles Beltrán y Liniers & Godoy Cruz & - & 1 & - & - & - & 1 \\
\hline Calles Tacuarí y 20 de Junio & Godoy Cruz & 1 & 3 & - & - & - & 4 \\
\hline Calle Olascoaga & Las Heras & - & - & - & 1 & - & 1 \\
\hline Ranchillos & Las Heras & 1 & - & - & - & - & 1 \\
\hline El Algarrobito & Lavalle & - & 1 & - & - & - & 1 \\
\hline
\end{tabular}


pasta (Falabella 1997; Sanhueza 1997). La variabilidad de patrones y familias de pasta se usó para evaluar la variabilidad tecnológica.

El análisis decorativo se realizó con el objetivo de definir las reglas que organizan el diseño, las técnicas y los colores, de este modo se evaluó la regularidad intra-categoría morfológica, inter-categorías e intersitios. El análisis de los diseños se llevó a cabo descomponiendo jerárquicamente las unidades básicas de diseño (Prieto 2005). Siguiendo a Lagiglia (1978) los atributos que nos permitieron definir la cerámica Viluco-inka son: a nivel de formas, aisana y aribaloide; a nivel decorativo, apéndices ornitomorfos, asa en opalillo y aletones en escudillas. Las jarras y keros de Agua Amarga fueron adscriptos a momentos de dominación inka por asociación contextual a vasijas Viluco-Inka. Todas las escudillas enteras analizadas presentan algún tipo de apéndice que permite adscribirlas al tipo Viluco-Inka.

\section{Resultados de los Análisis Cerámicos}

\section{Análisis morfométrico de vasijas enteras}

El estudio de las piezas enteras y reconstruidas $(n=40)$ permitió definir la presencia de cinco tipos cerámicos: jarra $(n=18)$; aisana o botella $(n=1)$; escudilla con apéndices $(n=9)$; kero con y sin asa $(\mathrm{n}=10)$; aribaloide $(\mathrm{n}=2)$. El análisis intra-vasija de las DS de todas las medidas tomadas permitió observar que todas las categorías presentan DS bajas, excepto los espesores que presentan las DS más altas, por lo que en términos generales definimos que hay regularidad en la manufactura. Comparando las categorías morfológicas, las vasijas más regulares son las aisanas y los aribaloides, seguidos por las escudillas y los keros, por otro lado, las jarras son las que presentan DS más altas (Tablas 2, 3 y 4).

En cuanto a los tamaños modales, definimos dos capacidades (volúmenes) para cada categoría morfológica, donde las medidas no se superpusieron. Las escudillas presentan las siguientes medidas: pequeñas con volúmenes entre 275 y 400 cc (Diám. Borde entre 139 y $149 \mathrm{~mm}$ ); grandes con volúmenes entre 750 y 1.100 cc (Diám. Borde entre 176 y $189 \mathrm{~mm}$ ); en la HTotal las medidas de ambos tamaños se superponen. En los keros las medidas son: pequeños con volúmenes entre 400 y $580 \mathrm{cc}$; grandes con volúmenes entre los 700 y 900 cc; en los Diám. Máximos y la HTotal las medidas se superponen. En el caso de las jarras: pequeñas con volúmenes

Tabla 2. Media y desviaciones estándar (DS) de escudillas.

Mean and standard deviation (SD) of escudillas.

\begin{tabular}{|c|c|c|c|c|c|c|c|}
\hline Sitio & Escudilla & $\begin{array}{c}\mathrm{H} \\
\text { Total } \\
(\mathrm{mm}) \\
\text { Media } \\
\text { DS } \\
\end{array}$ & $\begin{array}{c}\text { Diám. } \\
\text { Borde } \\
(\mathrm{mm}) \\
\text { Media } \\
\text { DS }\end{array}$ & $\begin{array}{l}\text { Diám. } \\
\text { Base } \\
(\mathrm{mm}) \\
\text { Media } \\
\text { DS } \\
\end{array}$ & $\begin{array}{c}\text { Espesor } \\
\text { Cuerpo } \\
\text { (mm) } \\
\text { Media } \\
\text { DS }\end{array}$ & $\begin{array}{c}\text { Espesor } \\
\text { Borde } \\
(\mathrm{mm}) \\
\text { Media } \\
\text { DS }\end{array}$ & $\begin{array}{l}\text { Vol. } \\
\text { (cc) }\end{array}$ \\
\hline \multirow{3}{*}{ Agua Amarga } & $\mathrm{n}^{\mathrm{o}} 10335$ & $\begin{array}{l}62 \\
2,95\end{array}$ & $\begin{array}{l}184 \\
2,291\end{array}$ & $\begin{array}{l}32 \\
2,629\end{array}$ & $\begin{array}{l}6,5 \\
1,11\end{array}$ & $\begin{array}{l}4,5 \\
0,386\end{array}$ & 750 \\
\hline & $\mathrm{n}^{\mathrm{o}} 10336$ & $\begin{array}{l}52 \\
1,414\end{array}$ & $\begin{array}{l}176 \\
2,31\end{array}$ & $\begin{array}{l}48,3 \\
2,363\end{array}$ & $\begin{array}{l}6,9 \\
0,25\end{array}$ & $\begin{array}{l}6 \\
0,356\end{array}$ & 750 \\
\hline & $\mathrm{n}^{\circ} 1219$ & $\begin{array}{l}66 \\
0,548 \\
\end{array}$ & $\begin{array}{l}189 \\
1,225 \\
\end{array}$ & $\begin{array}{l}53 \\
7,274 \\
\end{array}$ & $\begin{array}{l}5,6 \\
0,455 \\
\end{array}$ & $\begin{array}{l}4,4 \\
0,858 \\
\end{array}$ & 1.000 \\
\hline \multirow{2}{*}{ Viluco } & $\mathrm{n}^{\circ} 199$ & $\begin{array}{l}77 \\
0,548\end{array}$ & $\begin{array}{l}182 \\
2,944\end{array}$ & $\begin{array}{l}39,3 \\
0,977\end{array}$ & $\begin{array}{l}6,9 \\
0,420\end{array}$ & $\begin{array}{l}5,7 \\
0,469\end{array}$ & 1.100 \\
\hline & $\mathrm{n}^{\circ} 211$ & $\begin{array}{l}85 \\
1,893 \\
\end{array}$ & $\begin{array}{r}184 \\
1,414 \\
\end{array}$ & $\begin{array}{l}95 \\
0,577 \\
\end{array}$ & $\begin{array}{l}6,8 \\
0,208 \\
\end{array}$ & $\begin{array}{l}4,4 \\
0,191 \\
\end{array}$ & 800 \\
\hline Barrancas & $\mathrm{n}^{\circ} 8077$ & $\begin{array}{l}52 \\
2,168 \\
\end{array}$ & $\begin{array}{r}146 \\
1,14 \\
\end{array}$ & $\begin{array}{c}38,3 \\
2,363 \\
\end{array}$ & $\begin{array}{l}7,6 \\
0,415 \\
\end{array}$ & $\begin{array}{l}5,1 \\
0,454 \\
\end{array}$ & 400 \\
\hline Tacuarí y 20 de Junio & $n^{\circ} 3993$ & $\begin{array}{l}51 \\
0,577 \\
\end{array}$ & $\begin{array}{r}139 \\
1.915 \\
\end{array}$ & $\begin{array}{l}39 \\
0,957 \\
\end{array}$ & $\begin{array}{l}7 \\
0,408 \\
\end{array}$ & $\begin{array}{l}5,9 \\
0,096 \\
\end{array}$ & 400 \\
\hline Capiz I & E $16-1$ & $\begin{array}{c}54 \\
0,548 \\
\end{array}$ & $\begin{array}{l}149 \\
1,155 \\
\end{array}$ & $\begin{array}{l}43 \\
0,577 \\
\end{array}$ & $\begin{array}{l}5,8 \\
1,19 \\
\end{array}$ & $\begin{array}{l}4,2 \\
0,245 \\
\end{array}$ & 380 \\
\hline Ranchillos & $\mathrm{n}^{\circ} 1212$ & $\begin{array}{l}29 \\
0,957\end{array}$ & $\begin{array}{l}161 \\
1,154\end{array}$ & - & $\begin{array}{l}5 \\
0,707\end{array}$ & $\begin{array}{l}4,7 \\
1,289\end{array}$ & 275 \\
\hline
\end{tabular}




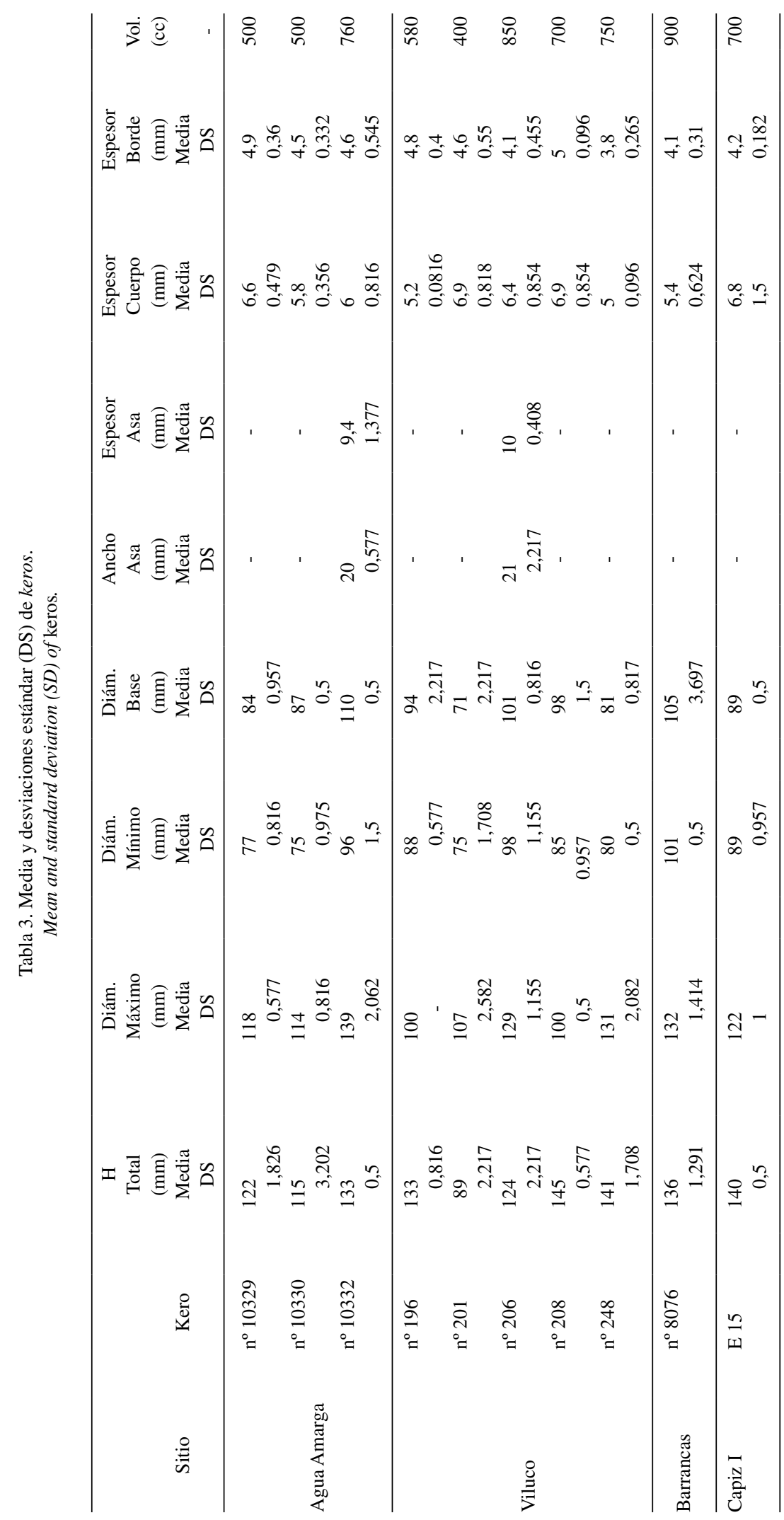




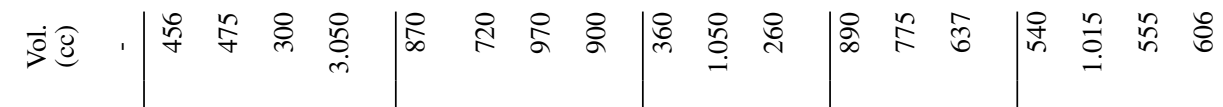

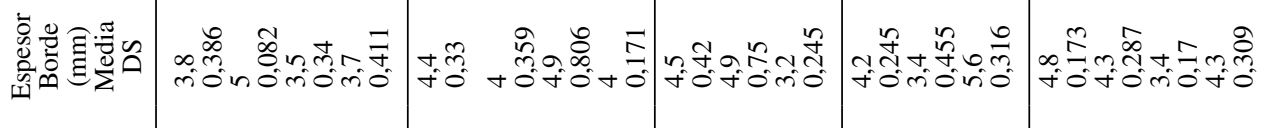

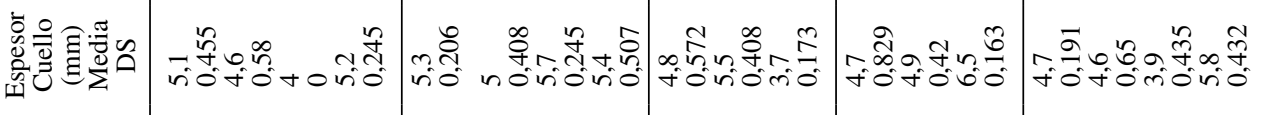

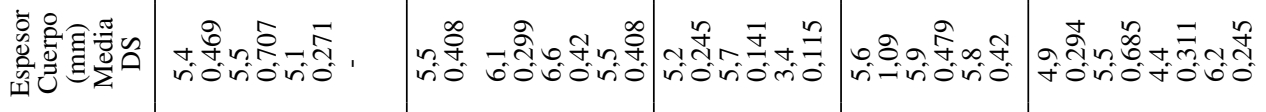

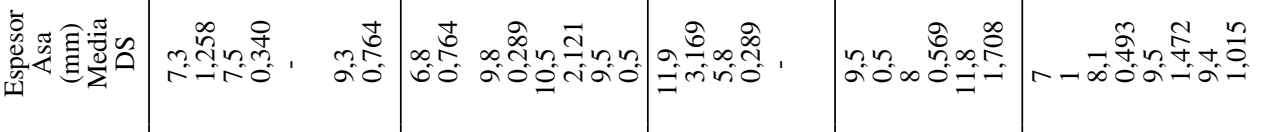

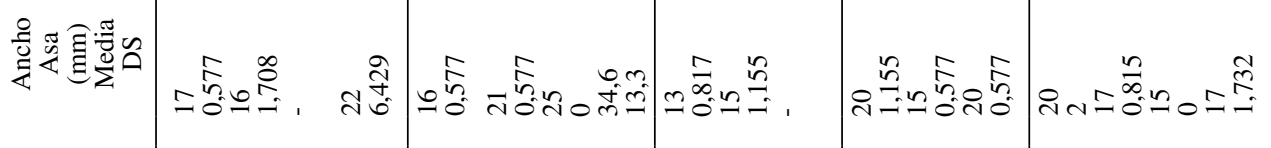

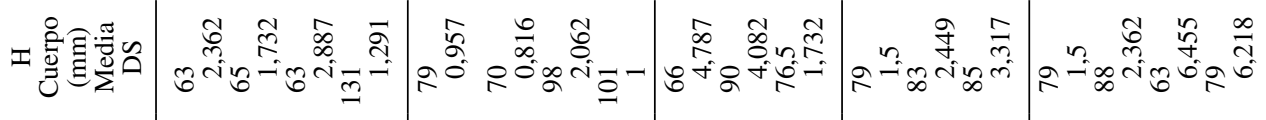

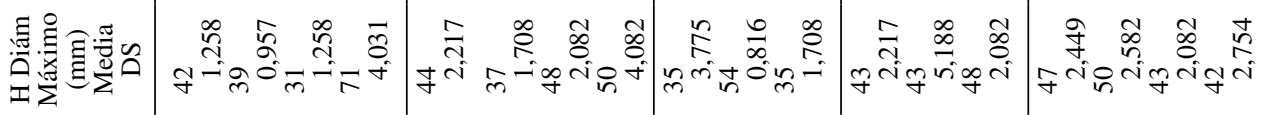

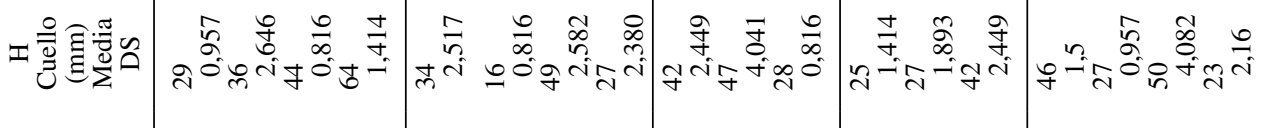

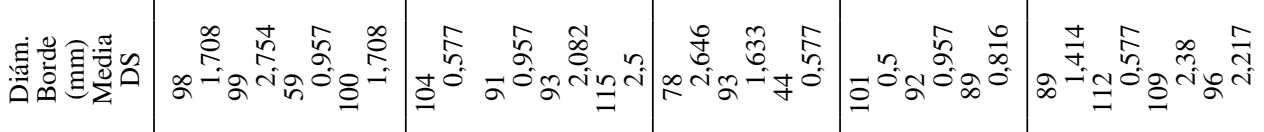

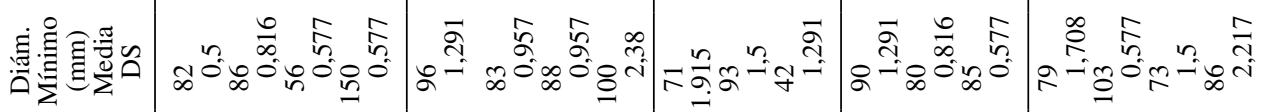

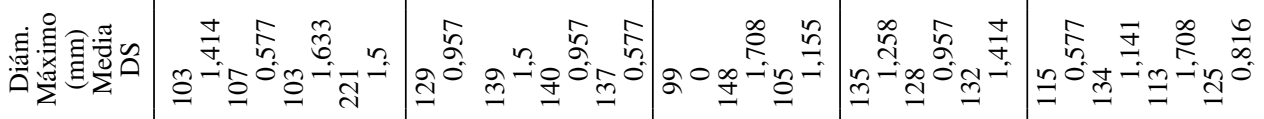

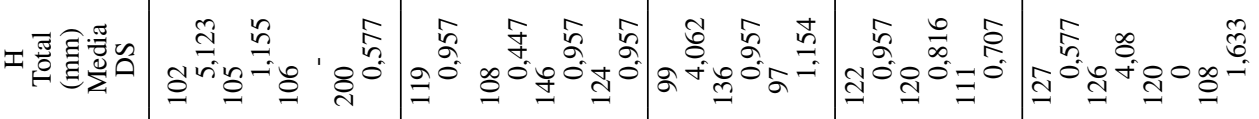

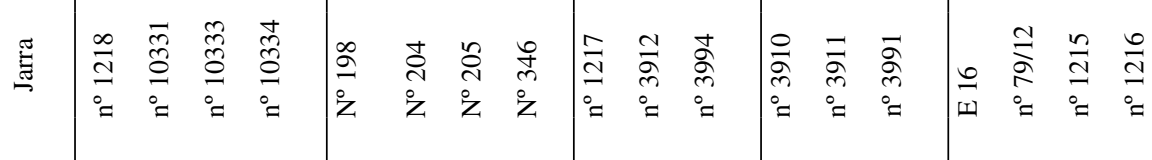

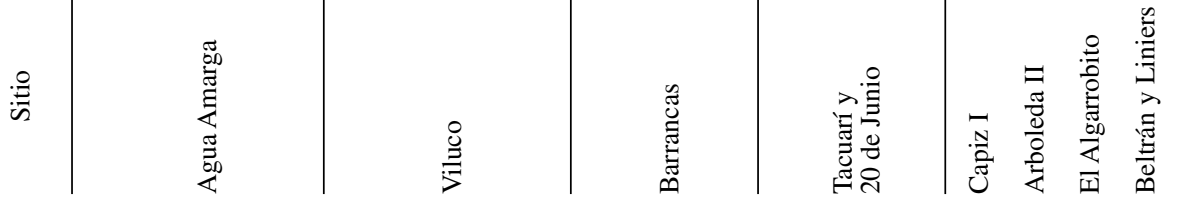


entre 260 y 1.050 cc, la HTotal oscila entre los 97 y $146 \mathrm{~mm}$; un solo ejemplar grande con un volumen de 3.050 cc y HTotal de 200 mm; los Diám. Borde se superponen entre ambos tamaños.

El análisis intra-categoría se realizó con la comparación de las DS a nivel intra e inter-sitios, lo cual permitió definir dos situaciones distintas en la cerámica funeraria: por un lado los keros, escudillas y jarras provenientes de los sitios Barrancas,
Viluco y Tacuarí y 20 de Junio (valle de Mendoza) presentan variabilidad morfométrica, mientras que las mismas categorías morfológicas del sitio Agua Amarga (valle de Uco) presentan regularidad en cada uno de los niveles de análisis (intra-vasija, intra-categoría) (Tablas 2, 3 y 4), lo que también se observó a nivel decorativo (Figura 2), por lo que definimos la existencia de vasijas pares. Las jarras pares de Agua Amarga ( $n^{\circ} 1218$ y 10331)

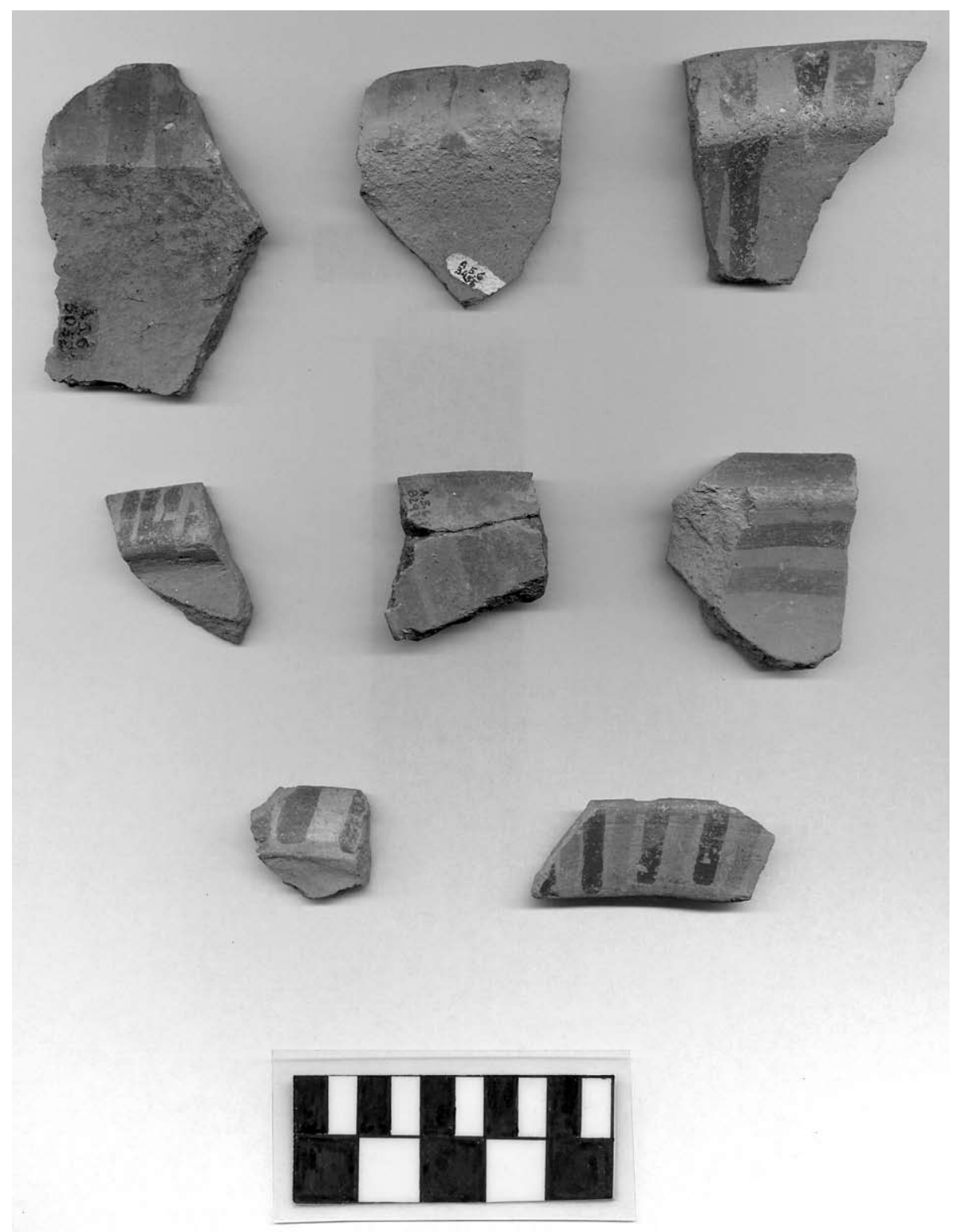

Figura 2. Fragmentos de bordes de platos (Prieto 2005: Lámina LI).

Plate rim fragments (Prieto 2005: Lámina LI). 
presentan similitud métrica en volumen, HTotal, Diám. Máximo, Diám. Mímino, Diám. Borde, HCuerpo, ancho y espesor del asa, espesor del cuerpo. Entre las escudillas pares $\left(\mathrm{n}^{\circ} 1219\right.$ y 10335) hay similitud en las medidas de HTotal y Diám. Borde, mientras que los volúmenes son disímiles. La escudilla 10336 tiene el mismo volumen que la $\mathrm{n}^{\mathrm{o}} 10335$ (750 cc). En los keros pares ( $\mathrm{n}^{\mathrm{o}} 10329$ y 10330) las medidas similares son volumen, HTotal, Diám. Máximo, Diám. Mínimo, Diám. Base. En los otros sitios que tienen buena representación de estas vasijas no observamos esta situación, aunque destacan las tendencias métricas parecidas que hay entre las jarras 198 y 346 del sitio Viluco.

\section{Análisis morfométrico de los fragmentos de RSF}

A partir del estudio de las vasijas enteras se evaluó la posibilidad de discriminar categorías morfológicas y tamaños entre los fragmentos de RSF. El resultado del análisis distribucional de medidas en las vasijas enteras posibilitó determinar que algunas servían para definir la categoría morfológica en los fragmentos. Entre las escudillas fue el Diám. Borde y los atributos cualitativos son los bordes rectos, los apéndices, de cabecitas ornitomorfas y aletones, de asas planas en los bordes, la ausencia de cuello y la superficie interior pulida (sin descartar alisados) y decorada con motivos pintados. Entre las jarras sirven la altura del cuello (HCuello) y el espesor del cuello para diferenciarlas de los aribaloides. Los atributos cualitativos que sirven para discriminar esta categoría son bordes ligeramente evertidos, cuellos, uniones cuello-cuerpo y asas con incisiones y/o pastillaje. En los aribaloides sólo tuvimos acceso a dos ejemplares, por lo que los cálculos son estimativos, las medidas que sirven son Diám. Borde, Diám. Mínimo, Diám. Máximo, Diám. Base, HTotal y el espesor del cuello para diferenciarlos de las jarras. Entre los atributos cualitativos destaca el punto angular que produce un corte entre el borde y el cuello ( $\left.n^{\circ} 10348\right)$, el punto angular que separa el sector inferior del cuerpo y la base cóncava. Entre los keros la única medida clara es el Diám. Base, mientras que los atributos cualitativos tienen mucha importancia, como por ejemplo el punto angular que marca el fin del cuerpo y el comienzo de la base y las paredes rectas o ligeramente curvadas?

Los resultados se aplicaron a los fragmentos de RSF, diferenciándose cualitativa y cuantitativamente sólo dos categorías morfológicas: escudillas $(n=360)$ y jarras $(n=79)^{10}$. El escaso tamaño de los fragmentos no permitió definir aspectos sobre la regularidad y/o variabilidad, pero sí fue posible potenciar la información a partir de la comparación de este contexto doméstico con la evidencia funeraria, observándose medidas mayores en las vasijas domésticas.

Entre las escudillas enteras los coeficientes de correlación obtenidos permiten definir que la predicción es posible a partir del Diám. Borde y HTotal (Tabla 5). Entre los fragmentos de escudilla se pudieron realizar 18 predicciones y destaca que en doce casos se definieron volúmenes muy grandes (entre $982 \mathrm{cc}$ a $2.372 \mathrm{cc}$ ). En el caso de las jarras completas y en base a los coeficientes obtenidos, las predicciones de capacidad se pueden hacer con el Diám. Máximo, el Diám. Mínimo y la altura del diámetro máximo (H Diám. Máximo) (Tabla 6). Entre los fragmentos de jarra la predicción se hizo en 21 casos, seis de ellas presentaron volúmenes mayores que los encontrados en los contextos funerarios (entre $3.269 \mathrm{cc}$ y $5.564 \mathrm{cc}$ ). El resultado de estas predicciones permiten confirmar que la inclusión en los análisis de fragmentos provenientes de contextos domésticos sirven para potenciar la información de los conjuntos cerámicos y disminuyen el sesgo producido por las tipologías basadas sólo en evidencia funeraria. En base a la evidencia postulamos que

Tabla 5. Coeficiente de correlación y ecuaciones de regresión de volumen de las escudillas.

Correlation coefficient and regression equation for bowl volumes.

\begin{tabular}{lcccc}
\hline \multicolumn{4}{c}{ Escudilla $(Y=$ Volumen total $) \mathrm{Y}=\mathrm{a}+\mathrm{b} \mathrm{X}$} \\
\hline $\mathrm{X}$ & $\mathrm{r}$ & $\mathrm{a}$ & $\mathrm{b}$ & $\mathrm{n}$ \\
Diám. Borde & 0,876 & $-1859,065$ & 14,850 & 9 \\
H Total & 0,769 & $-180,094$ & 14,078 & 9 \\
\hline
\end{tabular}

Tabla 6. Coeficiente de correlación y ecuaciones de regresión de volumen de las jarras. Correlation coefficient and regression equation for jar volumes.

Jarra (Y=Volumen total $) \mathrm{Y}=\mathrm{a}+\mathrm{b} \mathrm{X}$

$\begin{array}{lcccc}\text { X } & \mathrm{r} & \mathrm{a} & \mathrm{b} & \mathrm{n} \\ \text { Diám. Máximo } & 0,968 & -1959,262 & 21,485 & 18 \\ \text { Diám. Mínimo } & 0,902 & 1380,654 & 25,524 & 18 \\ \text { H Diám. Máximo } & 0,895 & -1937,157 & 61,469 & 18\end{array}$


las mayores capacidades detectadas entre las escudillas y las jarras de RSF estarían relacionadas con funciones domésticas, mientras que en los contextos funerarios se encuentra representado sólo una parte del conjunto alfarero. Cabe destacar que los análisis funcionales nos permitieron definir que escudillas y jarras cumplieron una doble función: doméstica y funeraria (Prieto 2005). Tampoco desestimamos la posibilidad que con el ingreso español se introdujeran tamaños mayores en las vasijas. Por otro lado, la presencia de escasos apéndices de bordes entre las escudillas de RSF (2,5\%), a diferencia de las escudillas enteras, indicaría que este tipo está escasamente representado en RSF, lo que puede tener una explicación cronológica, ya que el contexto es de período de contacto hispano-indígena; por otra parte, también puede deberse a aspectos funcionales ligados al contexto doméstico.

En este conjunto fragmentario también se definió un nuevo tipo cerámico Viluco: platos $(\mathrm{n}=12)$. Estos tienen la particularidad de presentar atributos morfológicos característicos de los platos españoles de mayólica, pero las pastas, decoraciones y tratamientos de superficie remiten a la tradición Viluco (Figura 3). Proponemos que estas formas serían producto del contacto hispano-indígena, ya que uno
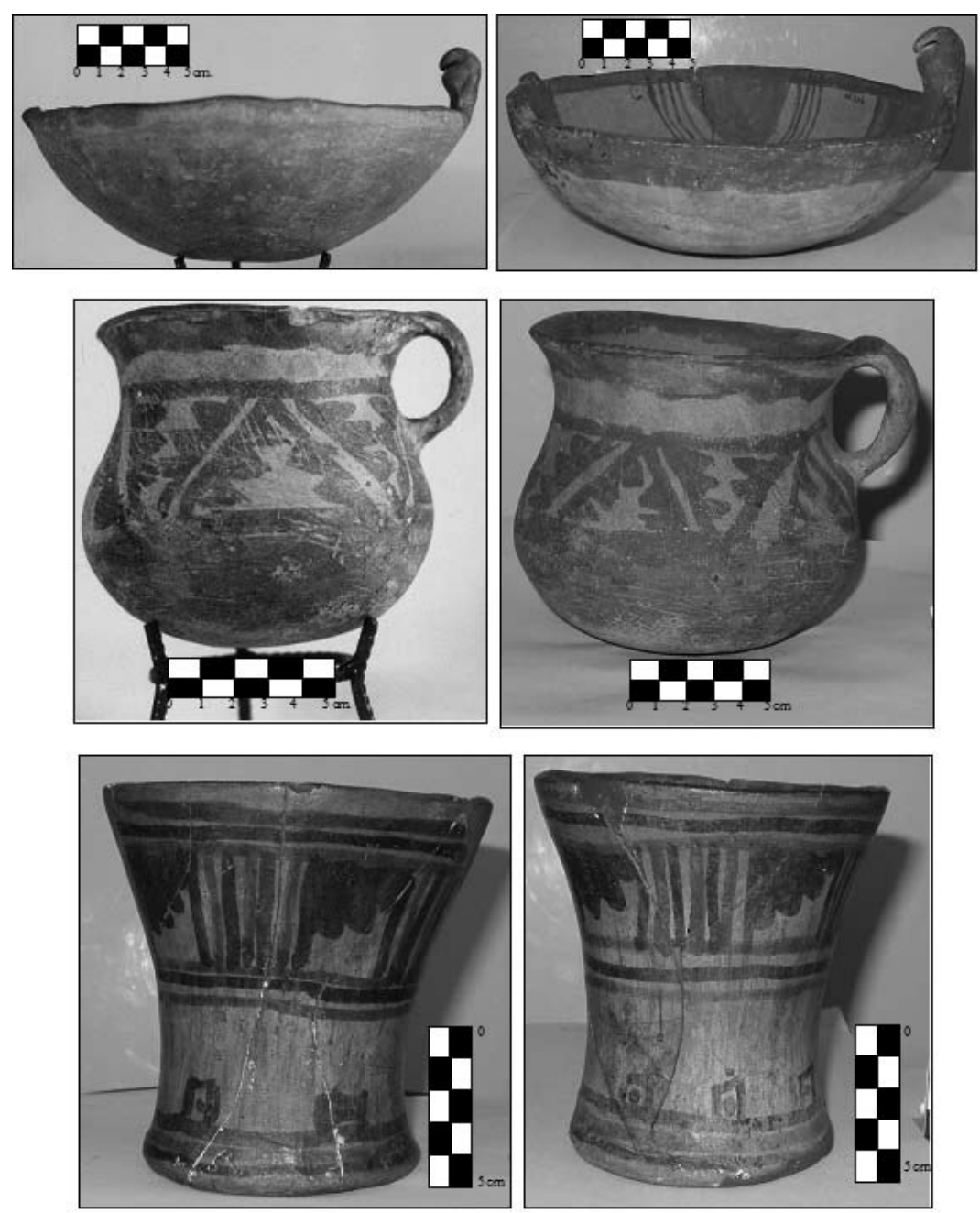

Figura 3. Vasijas pares del sitio Agua Amarga. Escudillas ornitomorfas $\mathrm{n}^{\circ} 1219 \mathrm{y} \mathrm{n}^{\circ} 1035$; jarras $\mathrm{n}^{\circ} 1218 \mathrm{y} \mathrm{n}^{\circ} 10331$; keros $\mathrm{n}^{\circ} 10329 \mathrm{y} \mathrm{n}^{\circ} 10330$.

Pair vessels of Agua Amarga site. Ornithomorphic bowls $n^{\circ} 1219$ and $n^{\circ} 1035$; jar $n^{\circ} 1218$ and $n^{\circ} 10331$; $\operatorname{keros} n^{\circ} 10329$ and $n^{\circ} 10330$. 
de los fragmentos presenta huellas de confección en torno, tecnología que fue incorporada después de la llegada de los españoles.

\section{Análisis de pastas}

Estos estudios se acotaron casi exclusivamente al material fragmentario de RSF, ya que gran parte de las vasijas enteras y reconstruidas no presentaron fracturas frescas para realizar la observación y sólo se pudo realizar en dos vasijas del sitio Capiz I. Entre los fragmentos (RSF) se observó gran variabilidad, un total de 39 patrones de pasta agrupados en 10 familias (Tabla 7). Destacamos que entre éstos se encontraron patrones definidos para otros sitios de la provincia: el patrón Cc fue descrito para el cementerio de Capiz I (Prieto y Durán 2007); los patrones de pasta $2,10,20,26,50$ fueron definidos para diversos sitios de la Planicie $\mathrm{NE}^{11}$. Esto nos lleva a proponer una amplia dispersión de los patrones de pasta en gran parte del área Viluco (norte y centro de Mendoza: planicie NE, valle de Mendoza y valle de Uco).

Un reciente estudio petrográfico realizado con las pastas de la cerámica Viluco, los platos de manufactura Viluco y la cerámica colonial de tradición europea permitió definir que la elaboración de los platos no habría implicado un cambio tecnológico en el tratamiento de las pastas, manteniéndose la tradición Viluco (especialmente el procesamiento), mientras que la innovación estuvo en la incorporación de nuevas formas y del torno (Prieto et al. 2006).

Tabla 7. Patrones y familias de pasta de Ruinas de San Francisco (RSF).

Patterns and paste types for Ruinas de San Francisco $(R S F)$.

\begin{tabular}{lccccccc}
\hline \multicolumn{1}{c}{ Familia } & \multicolumn{7}{c}{ Patrones } \\
\hline XI & $1 \mathrm{cr}$ & $1 \mathrm{vr}$ & $2 \mathrm{r}$ & $3 \mathrm{r}$ & 2 & Gc & 26 \\
XII & $10 \mathrm{r}$ & $12 \mathrm{ar}$ & $1 \mathrm{hr}$ & $4 \mathrm{r}$ & $7 \mathrm{r}$ & $26 \mathrm{r}$ & $9 \mathrm{r}$ \\
XIII & $11 \mathrm{r}$ & & & & & & \\
XIV & $12 \mathrm{r}$ & $22 \mathrm{r}$ & $\mathrm{Cc}$ & 10 & & & \\
XV & $19 \mathrm{r}$ & $15 \mathrm{r}$ & 20 & 50 & & & \\
XVII & $5 \mathrm{r}$ & $5 \mathrm{ar}$ & $14 \mathrm{r}$ & $5 \mathrm{rr}$ & & & \\
XIX & $21 \mathrm{r}$ & & & & & \\
XX & $1 \mathrm{br}$ & $27 \mathrm{r}$ & $17 \mathrm{r}$ & $8 \mathrm{r}$ & $8 \mathrm{rr}$ & \\
XXI & $6 \mathrm{r}$ & $23 \mathrm{r}$ & & & & \\
XXII & $16 \mathrm{r}$ & $6 \mathrm{ar}$ & $18 \mathrm{r}$ & & & \\
Sin familia & $25 \mathrm{r}$ & & & & & \\
\hline
\end{tabular}

\section{Análisis de decoración}

Los diseños son mayoritariamente geométricos y en menor medida se representaron figuras que pueden describirse como "soles", pero todas fueron analizadas descomponiéndolas en unidades mínimas de análisis. Con el análisis de las unidades de diseño en las vasijas enteras observamos que las más complejas se encontraban en jarras y keros, además de determinar que estaban organizadas a nivel y de áreas decoradas diferencialmente. También se detectó la existencia de esqueletos modeladores (constructivo y rítmico), además de la organización de los colores. En este último caso, la decoración policroma se organiza a partir de la alternación de colores "claros" (rojo o violáceo) y "oscuros" (negro, marrón o marrón oscuro). En la evaluación de la variabilidad de la decoración definimos dos situaciones: los sitios de Barrancas, Viluco y Tacuarí y 20 de junio, no presentan regularidad a nivel intra-categoría morfológica; por el contrario, el sitio de Agua Amarga presentó gran regularidad en las vasijas gemelas: las escudillas $\left(\mathrm{n}^{\circ} 1219\right.$ y 10335$)$ presentan el motivo modelado de cabecitas ornitomorfas con cascabel; las jarras $\left(\mathrm{n}^{\mathrm{o}} 1218\right.$ y 10331$)$ presentan los mismos motivos pintados y configuración; los keros $\left(\mathrm{n}^{\mathrm{o}} 10329\right.$ y 10330) tienen los mismos motivos pintados y configuración.

Entre los fragmentos se observó una mayor cantidad de unidades decorativas que las definidas en las vasijas enteras, pero debido al pequeño tamaño de los fragmentos no se pudo hacer reconstrucciones como se hizo a nivel morfométrico. También determinamos que algunos motivos que no aparecían entre las escudillas enteras, sí se encontraron en los fragmentos de escudillas de RSF, información que potencia y aumenta la obtenida de los contextos funerarios.

La decoración de los platos no se pudo comparar con datos antecedentes, pero proponemos la resistencia al cambio decorativo, debido a la persistencia de motivos Viluco en el marco de la introducción de nuevas formas y tecnología (torno). En este sentido, adherimos a la propuesta sobre el concepto de tradición tecnológica como el aspecto más resistente al cambio, pero también a la posibilidad que aspectos como la forma y la decoración se mantuviera en el tiempo, tal vez sostenido por factores sociales e ideológicos (Sanhueza 1997). 


\section{Discusión y Conclusiones}

El problema abordado en este estudio se centra en un complejo y corto período de tiempo que incluye las poblaciones Viluco asentadas en el norte y centro de la provincia de Mendoza durante el período de contacto Viluco-Inka, hispano-indígena y colonial temprano, segunda mitad del siglo XV y mediados del XVII. La integración de la información de las vasijas enteras con los fragmentos permitió potenciar ambas fuentes de información y elaborar una visión integral del conjunto cerámico Viluco, interrelacionando la información de contextos funerarios y doméstico. El problema lo hemos abordado desde la perspectiva de la variabilidad cerámica, cuyo estudio permite introducirse en los procesos de producción alfarera de un grupo, objetivo central de este estudio.

Los resultados de los análisis morfométrico y decorativo han permitido explorar algunos aspectos de la producción cerámica Viluco, y con ello obtener una ajustada caracterización alfarera. El análisis de las vasijas enteras permitió definir dos situaciones. En primer lugar, el conjunto alfarero Viluco-Inka del sitio Agua Amarga presenta regularidad morfológica y decorativa a nivel intra-vasija (aribaloide y aisana) y la presencia de vasijas gemelas (escudillas ornitomorfas, jarras y keros). Esta evidencia nos permite proponer una estandarización en la producción cerámica que estaría directamente relacionada con la propuesta de un enclave Diaguita-Inka en el área (Ots 2007). Por otra parte, los otros sitios que presentan varias vasijas, como Barrancas, Viluco y Tacuarí y 20 de Junio, y que tienen vasijas Viluco y Viluco-Inka, no evidencian regularidad intra-categoría y la regularidad intra-vasija no es tan clara como en las vasijas de Agua Amarga. Esta situación y su origen debe ser explorada con mayor profundidad, aunque al respecto podemos mencionar que en el valle de Mendoza no se ha registrado arqueológicamente -aunque sí etnohistóricamente- asentamientos de poblaciones asentadas por el inka.

Por otra parte, los resultados obtenidos en el análisis tecnológico (pasta) de los fragmentos de RSF permite tener una primera aproximación a la producción cerámica de las vasijas representadas en un sitio doméstico. Hemos interpretado esta variabilidad como resultado de una producción descentralizada posterior a la dominación inka. Por otra parte, la amplia dispersión de estos patrones de pasta y los definidos para otros sitios, y que abarca desde el valle de Uco (centro de la provincia) hasta el valle de Mendoza y noreste provincial, permitiría indagar aspectos como la movilidad y/o intercambio, situación que ha sido descrita a partir de la información documental (Parisii 1998; Prieto 2000).

Todos estos resultados apuntan a reflexionar sobre algunos de los cambios que se suscitaron a partir de la incorporación de los grupos locales a los dominios Inka y español. Los antecedentes arqueológicos y etnohistóricos entregan algunos indicios que apoyan nuestras propuestas, y sirven de base para seguir indagando otros caminos de interpretación. Un aspecto fundamental es comprender cómo pudo ser la producción cerámica en momentos de dominación Inka en el valle de Uco; tal vez pudo darse en el marco de un control de la manufactura con una alta inversión por parte del sistema estatal (D'Altroy et al. 1994). Particularmente lo que observamos en Agua Amarga es la producción de vasijas Viluco-Inka estandarizadas, lo cual pudo ser funcional a las relaciones asimétricas que se dieron entre los grupos locales, a partir de la relación diferencial que sostuvieron con la población Inka que se asentó en el área. Esta situación se ha postulado desde la etnohistoria para Mendoza (Parisii 1998) con la jerarquización de las parcialidades detectada a nivel documental y de modo más amplio a partir de los estudios sobre producción cerámica en distintos lugares de la dominación Inka (D'Altroy et al. 1994). Arqueológicamente, se ha definido la presencia de alfareros locales manufacturando en el valle de Uspallata (valle intercordillerano) en el tambo de Tambillos (Bárcena y Román 1990).

Respecto a la producción de un nuevo tipo, los platos, en el sitio RSF (valle de Mendoza), permite proponer que la población Viluco de este sector fue sensible a los rápidos y profundos cambios que se suscitaron con la incorporación del área al imperio español, especialmente si incorporaron una nueva tecnología como el torno. En cuanto al proceso de producción, la introducción del torno y de nuevas formas pudo implicar cierto grado de control de los artesanos por parte de la nueva población dominante, los españoles. De todos modos, el mantenimiento de la decoración Viluco y la tecnología de las pastas podría considerarse como una resistencia al cambio y relacionarse con aspectos ideológicos o sociales de una población que decreció considerablemente debido a la encomienda.

Agradecimientos: nuestros agradecimientos al trabajo desinteresado y anónimo de los evaluadores. 


\section{Referencias Citadas}

Bárcena, J.

1994 (1991-1992) Datos e interpretación del registro documental sobre la dominación incaica en Cuyo. Xama 4-5:11-49.

1998 Arqueología de Mendoza: Las Dataciones Absolutas y sus Alcances. EDIUNC, Mendoza.

Bárcena J. y A. Román

1990 [1986-1987] Funcionalidad diferencial de las estructuras del tambos de Tambillos: resultados de la excavación de los recintos 1 y 2 de la Unidad A del Sector III. Anales de Arqueología y Etnología 41-42:7-81.

Chiavazza, $\mathrm{H}$.

1995 Estudios Arqueológicos en el Sitio "Rincón de Los Helados" ("RH"). Ocupación Multicomponente en Noreste de Pampa de Canota - Departamento de Las Heras, Provincia de Mendoza, República Argentina. Tesis de Licenciatura, Facultad de Filosofía y Letras, Universidad Nacional de Cuyo, Mendoza.

2007 Cambio Ambiental y Sistemas de Asentamiento en el Árido Normendocino. Arqueología en torno a los Paleocauces del Río Mendoza. Tesis doctoral, Facultad de Ciencias Naturales y Museo, Universidad Nacional de La Plata, La Plata.

Chiavazza, H. y C. Prieto

2001 Arqueología en el predio jesuita de la antigua ciudad de Mendoza-Centro Oeste de Argentina. CD-ROM Multimedia Didáctico, X Congreso Uruguayo de Arqueología, editado por L. Beovide, I. Barreto y C. Curbelo, Montevideo.

Costin, C.

1991 Craft specialization: issues in defining, documenting, and explaining the organization of production. Archaeological Method and Theory, editado por M. Schiffer, Vol. 3, pp. 1-56. University of Arizona Press, Tucson.

D'altroy, T., A. Lorandi y V. Williams 1994 Producción y uso de cerámica en la economía política Inka. Tecnología y Organización de la Cerámica Prehispánica en Los Andes, editado por I. Shimada, pp. 395-441. Fondo Editorial, Perú.

Durán, V. y P. Novellino

2003 Vida y muerte en la frontera del Imperio Español. Estudios arqueológicos y bioantropológicos en un cementerio indígena post-contacto del Centro Oeste de Argentina. Anales de Arqueología y Etnología 54-55:115-164.

Falabella, F.

1997 El estudio de la cerámica Aconcagua en Chile central: una evaluación metodológica. Actas del XIV Congreso Nacional de Arqueología Chilena Contribución Arqueológica Tomo 1: 427-458.

Falabella, F., A. Deza, A. Román y E. Almendras

1993 Alfarería Llolleo: un enfoque funcional. Actas XII Congreso de Arqueología Chilena Boletín del Museo Regional de La Araucanía 4(2): 327-353.
García, A.

1999 [1997] Alcances del dominio inkaico en el extremo suroriental del Tawantinsuyu, Argentina. Chungara 29:195-208.

Lagiglia, $\mathrm{H}$.

1978 La Cultura de Viluco del Centro Oeste Argentino. Revista del Museo de Historia Natural III (1-4):227-265.

Michieli, C.

1983 Los Huarpes Protohistóricos. Imprenta de la Universidad Nacional de San Juan, San Juan.

1998 Aproximaciones a la identificación de una cerámica indígena posthispánica del sur de San Juan. Publicaciones 22: 55-76, San Juan.

Ots, M.J.

2007 Datos e interpretación sobre la dominación incaica del valle de Uco, Mendoza. Actas XVI Congreso Nacional de Arqueología Argentina II, pp. 479-485. Jujuy.

Parisii, M.

1994 [1991-1992] Algunos datos de las poblaciones prehispánicas del Norte y Centro Oeste de Mendoza y su relación con la dominación Inca del área. Xama 4-5:51-69.

1998 Hipótesis alternativas para el estudio del cambio social y la oposición entre las poblaciones indígenas de Mendoza (siglosXVI-XVIII). Хama 6-11:145-166.

Prieto C.

2005 Alfarería Viluco en el Norte y Centro de la Provincia de Mendoza (Argentina): Nuevas Perspectivas Analíticas. Memoria para optar al título de Arqueólogo, Facultad de Ciencias Sociales, Universidad de Chile, Santiago.

Prieto, C. y V. Durán

2007 Cementerio indígena de Capiz Alto (San Carlos Mendoza). Actas del XIV Congreso Nacional de Arqueología Argentina, pp. 227-236. Universidad Nacional de Rosario, Rosario.

Prieto. C., L. Puebla y B. Castro

2006 Estudios petrográficos de cerámica arqueológica y fuentes de materias primas. El cambio tecnológico en el período de contacto hispano-indígena, el caso Ruinas de San Francisco (Mendoza). Actas 3 Congreso Nacional de Arqueología Histórica, Rosario, en prensa.

Prieto, M. R.

2000 Formación y consolidación de una sociedad en un área marginal del Reino de Chile: la Provincia de Cuyo en el siglo XVII. Anales de Arqueología y Etnología 52-53.

Sacchero, P. y A. García

1991 Una estación trasandina diaguita chilena. Actas del XI Congreso Nacional de Arqueología Chilena Tomo III, pp. 61-67. Santiago.

Sanhueza, L.

1997 Patrón cerámico: hacia la definición de un concepto operativo. Actas del XIV Congreso Nacional de Arqueología Chilena Contribución Arqueológica Tomo I, pp. 243-258.

\section{Notas}

1 Fechados TL del cementerio de Viluco: $1.440 \pm 60$ años d.C. (UCTL-486) (cerámica gris negra), 1.540 \pm 50 años d.C. (UCTL-487) (cerámica decorada Viluco); fechados del predio del Cabildo de Mendoza: 1.420 \pm 60 d.C. (UCTL-326), $1.470 \pm 50$ d.C. (UCTL-310), ambos en cerámica Viluco decorada (Bárcena 1998). Fechados de Capiz I: 450 \pm 45 a.p. - 1.550 d.C. (UCTL 1292) y $380 \pm 40$ a.p. - 1.615 d.C. (UCTL 1291) (Durán y Novellino 2003).

2 No existen pruebas documentales que avalen la existencia de señoríos estructurados, con autoridades duales y orga- 
nización en la explotación de recursos naturales (Parisii 1994).

3 Localizado en el sustrato del sector del crucero del templo actualmente en ruinas, entre los 235 y $240 \mathrm{~cm}$ de profundidad aproximadamente.

4 Los escasos contextos domésticos Viluco se encuentran altamente disturbados, especialmente en el valle de Mendoza.

5 Fueron relevadas de siguientes colecciones: Museo de Arqueología y Etnología, Facultad de Filosofía y Letras, Universidad Nacional de Cuyo; Museo de Historia Natural y Ciencias Antropológicas "Juan Cornelio Moyano"; Museo Municipal de Historia Natural de San Rafael.

6 Los trabajos son de Falabella (Falabella et al. 1993; Falabella 1997), Sanhueza (1997), para las sociedades del Agroalfarero Temprano, Intermedio Tardío y Tardío de Chile Central.

7 Se tomaron entre 4 y 5 medidas para cada atributo. En las tablas la altura se abrevia como $\mathrm{H}$, el diámetro como Diám. y el volumen Vol. Las medidas tomadas fueron: alto total, alto del cuerpo, alto del cuello, diámetro máximo, diámetro mínimo, diámetro de borde, diámetro unión cuello-cuerpo, diámetro de base, alto del diámetro máximo, alto del diámetro mínimo, alto unión cuello-cuerpo, alto superior del asa, alto inferior del asa, espesor del asa, largo del asa, ancho del asa, espesor de borde, espesor de cuello, espesor de cuerpo, espesor de base. También se midieron los volúmenes del cuello y del cuerpo, y el volumen total.

8 La identificación de los patrones de pasta se realizó en base a una nomenclatura basada en números y letras. Se detallan en la Tabla 7.

$9 \quad$ Las asas de jarras, keros y aribaloides presentan medidas (ancho y espesor) que se superponen, por lo que no sirven para ser usadas para discriminar categorías morfológicas en conjuntos fragmentarios.

10 Los resultados corresponden a los fragmentos sometidos al proceso de ensamblaje y a una evaluación para definir la probabilidad de correspondencia a una misma pieza.

11 Estos datos corresponden a la tesis doctoral de Horacio Chiavazza (2007). 